\title{
Impacto das pesquisas do Programa de Apoio ao Desenvolvimento Institucional do Sistema Único de Saúde
}

\author{
Impact of research on the Support Program for the Institutional \\ Development of the SUS (Unified Health System)
}

Luciana Hentzy Moraes', Antonia Angulo-Tuestaㄹ, Silvana Schwerz Funghetto ${ }^{\mathbf{2}}$, Tania Cristina Morais Santa Barbara Rehem

DOI: $10.1590 / 0103-11042019 S 205$

RESUMO Mensurar o impacto do investimento em pesquisas contribui para a compreensão do alcance dos resultados nos sistemas de saúde e para o direcionamento de recursos para áreas prioritárias. Este estudo avaliou o impacto na dimensão 'avanços no conhecimento' produzido pelas pesquisas em saúde financiadas pelo Programa de Apoio ao Desenvolvimento Institucional do Sistema Único de Saúde (Proadi-SUS), no período de 2009 a 2014, no Brasil. Trata-se de pesquisa avaliativa, que utilizou o modelo adaptado de avaliação de pesquisas em saúde da Canadian Academy of Health Sciences (CAHS), a partir de análise documental dos registros de acompanhamento de projetos e relatórios de prestação de contas apresentados pelas instituições executoras das pesquisas. Foram investidos R\$ 66,49 milhões em 46 pesquisas, em 12 áreas temáticas, distribuídas em cinco tipos de estudos, principalmente em cardiologia. Foram identificados produtos de 28 projetos (60,8\%). Observou-se potencial avanço do conhecimento no campo das doenças crônicas. A transferência dos conhecimentos gerados por essas pesquisas e o impacto do investimento nas categorias tomada de decisão informada e benefícios ao setor saúde não foram mensurados e permanecem como desafios para a efetiva avaliação do programa. Estudos que avaliem a aplicação das evidências produzidas na prática clínica e na gestão podem contribuir para a compreensão da medida do impacto das pesquisas financiadas pelo Proadi-SUS em outras dimensões.

1 Ministério da Saúde (MS)

- Brasília (DF), Brasil.

luhm1110@gmail.com

2 Universidade de Brasília (UnB), Faculdade de Ceilândia, Programa de Pós-graduação em Ciências e Tecnologias em Saúde - Brasília (DF), Brasil.

3 Universidade de Brasília (UnB), Faculdade de Saúde, Programa de Pós-Graduação em Saúde Coletiva - Brasília (DF), Brasil.
PALAVRAS-CHAVE Pesquisa em saúde. Avaliação da pesquisa em saúde. Financiamento da pesquisa. Saúde coletiva.

ABSTRACT Measuring the impact of investment in research can contribute to a better understanding of the achievement of results in health systems and to guide the management of resources for priority areas. This study sought to evaluate the impact of 'advances in knowledge' produced by research on health funded by the Support Program for the Institutional Development of the Unified Health System (Proadi-SUS) between 2009 and 2014 in Brazil. It is an evaluative investigation based on the institutional records of project monitoring and accountability reports presented by the research institutions. The impact analysis used some of the indicators proposed by the adapted health research evaluation model of the Canadian Academy of Health Sciences (CAHS). The global investments were of $R \$ 66.49$ million in 46 investigations and identified 12 subject areas, 
distributed in five types of studies. The main area was cardiology. The impact analysis identified the results of 28 projects (60.8\%). It was possible to observe potential advances in knowledge in the field of chronic non-infectious diseases. The transfer of knowledge generated by these surveys and the impact of investing on informed decision-making and health sector benefits have not been measured and remain challenges for an effective evaluation of the program. Studies that evaluate the use of evidence produced in clinical practice and management can help understand the impact of research funded by the Proadi-SUS in other dimensions.

KEYWORDS Health research. Health research evaluation. Research funding. Community Health.

\section{Introdução}

Os sistemas de pesquisa em saúde conectam a produção do conhecimento científico ao desenvolvimento, para o aprimoramento das condições de saúde e equidade. O valor atribuído à pesquisa possibilita que as evidências produzidas contribuam para melhorar as políticas públicas e o desempenho do sistema público de saúde. Recursos financeiros, humanos e materiais são investidos em pesquisas para prevenir e tratar doenças, mas apenas uma parcela de produtos desses investimentos se traduz em política e prática, contribuindo efetivamente para a melhoria nos indicadores de saúde, aprimoramento das condições de vida e de saúde da população. Nos últimos anos, o interesse em demonstrar os benefícios das pesquisas e a necessidade de justificar o investimento público têm incentivado instituições e agências de fomento a desenvolverem sistemas de avaliação capazes de mostrar o impacto das pesquisas à sociedade ${ }^{1}$. Mensurar o impacto desse investimento pode contribuir para aumentar a capacidade de financiar pesquisas estratégicas para o País, sobretudo em épocas de restrição orçamentária. Para compreender o caminho entre a pesquisa e o seu impacto em diferentes níveis da sociedade, sistemas de pesquisa em saúde, como os do Canadá, Austrália e do Reino Unido, estabeleceram modelos de avaliação que buscam mapear e identificar como os resultados das pesquisas se revertem em benefícios amplos, para além do meio acadêmico, como, por exemplo mudanças para a economia, a sociedade, os serviços públicos, o meio ambiente e a qualidade de vida ${ }^{2-4}$.

No Brasil, o Ministério da Saúde, por intermédio do Departamento de Ciência e Tecnologia (Decit), financia pesquisas por várias modalidades, entre as quais estão os programas de renúncia fiscal. Em 2009, foi instituído o Programa de Apoio ao Desenvolvimento Institucional do Sistema Único de Saúde (Proadi-SUS), concebido na perspectiva de apoiar a qualificação e o aperfeiçoamento do SUS. Esse programa decorre de um conjunto de normativos legais que possibilitou a certificação das entidades beneficentes de assistência social na saúde, consolidando a cooperação entre o Estado e o terceiro setor (filantropia) ${ }^{\mathbf{5}}$.

O Proadi funciona pela execução de projetos financiados por meio da renúncia fiscal concedida a seis hospitais de excelência, localizados nas regiões Sudeste e Sul ${ }^{6}$. Em dez anos de existência desse programa, recursos foram investidos em pesquisas que deveriam contribuir para a qualificação do SUS. No entanto, são escassas as avaliações sobre os resultados alcançados no campo da pesquisa.

Um dos pressupostos da avaliação de programas é fornecer elementos que permitam identificar evidências de pesquisa passíveis de incorporação ao sistema de saúde, visando ampliar o alcance dos resultados das pesquisas para a sociedade. A avaliação de programas 
busca oferecer elementos importantes que podem contribuir para aprimorar a capacidade de financiamento de pesquisas que ofereçam respostas aos problemas prioritários de saúde. Entende-se que as atividades de pesquisa envolvem incertezas quanto aos resultados, os quais nem sempre serão aplicáveis no curto e médio prazo ao sistema de saúde 7-9. $^{\text {. }}$

A relação entre o contexto do financiamento de pesquisas e os seus resultados e impactos é permeada por questões como: 1- Que contribuições e impactos são efetivamente atribuíveis aos resultados de uma determinada pesquisa? 2- Qual o impacto caso a pesquisa não fosse executada? 3- Qual o potencial impacto indesejável da pesquisa? 4- Qual o fluxo de conhecimento e a identificação de barreiras e facilitadores (quais, como, onde, por quem, para quem e por que) capazes de influenciar a transferência e absorção das evidências ao longo do tempo??

Dessa forma, este artigo avaliou os avanços no conhecimento provenientes das pesquisas desenvolvidas no âmbito do Proadi-SUS, no período de 2009 a 2014.

\section{Métodos}

Trata-se de avaliação do impacto das pesquisas desenvolvidas no âmbito do Proadi-SUS, no período de 2009 a 2014, por meio da dimensão avanços no conhecimento, uma das cinco dimensões do modelo adaptado de avaliação de pesquisa em saúde canadense da Canadian Academy of Health Sciences (CAHS) ${ }^{9,10}$.

A estrutura de avaliação CAHS combina uma abordagem multidimensional baseada em um modelo lógico a fim de compreender como o impacto é produzido, em cinco dimensões: 1 - avanços no conhecimento, 2 - capacidade de pesquisa, 3 - tomada de decisão informada, 4 - benefícios e melhorias para o setor saúde e para a saúde, 5 - benefícios econômicos e sociais. Esse modelo estabelece categorias, indicadores e métricas que permitem identificar o impacto em qualquer estágio e nível de aplicação (individual, institucional). Experiências no Canadá ${ }^{11}$, Espanha ${ }^{12}$, Irlanda ${ }^{13}$ e Brasil14 utilizaram essa metodologia.

Na dimensão avanços no conhecimento, objeto deste artigo, a escolha das categorias e indicadores considerou critérios de validade, replicabilidade, disponibilidade de dados, atribuição de dados às pesquisas financiadas, da seguinte forma:

1) Atividade da pesquisa: Contagem e percentual de publicações por tipo, referência a financiadores (patrocinadores).

2) Qualidade da pesquisa: número de artigos e de periódicos, ano de publicação, fator de impacto e avaliação Qualis Capes dos periódicos, número de citações e ranqueamento de artigos mais citados.

3) Disseminação de resultados: contagem de participação em eventos científicos e não científicos, modalidades de apresentação dos resultados da pesquisa.

A realização da pesquisa foi baseada em análise documental, tendo como fonte os registros de submissão e os relatórios de prestação de contas dos 46 projetos desenvolvidos pelos 6 hospitais de excelência participantes do Proadi-SUS entre 2009 e 2014. Foram avaliados os projetos no campo de avaliação de tecnologia em saúde e a pesquisa de interesse público, sendo excluídos os projetos que contemplavam exclusivamente a aquisição de equipamentos ou a estruturação de unidades e centros de pesquisa e os de capacitação de recursos humanos.

Inicialmente, realizou-se a caracterização das pesquisas considerando os recursos investidos, as áreas temáticas dos projetos, os tipos de estudos e a forma de condução das pesquisas. Em seguida, foi avaliado o impacto na dimensão avanços no conhecimento, por meio do mapeamento da produção científica e técnica (artigos científicos, resumos em anais de periódicos científicos, 
capítulos de livro) decorrente das pesquisas, referência aos financiadores, o fator de impacto dos periódicos, análise de citações e disseminação das pesquisas (apresentações em eventos científicos e eventos não científicos). Nessa etapa, foram consultadas as seguintes fontes: 1) relatórios finais de prestação de contas de contas dos projetos, para identificação da produção científica; 2) Plataforma Lattes (http://lattes.cnpq. br/), para verificação e confirmação das áreas de atuação, linhas de pesquisa dos pesquisadores, produções e participações em eventos científicos atribuídos às pesquisas; 3) Google acadêmico, para identificação de artigos e citações; 4) Base de dados Scopus para identificação de artigos e citações; 5) Base de dados SCImago que forneceu h-index e Journal Citation Report (JCR) dos periódicos; 6) Plataforma Sucupira para classificação Qualis Capes dos periódicos. A análise utilizou medidas descritivas para a caracterização das pesquisas e produtos gerados.

\section{Resultados}

\section{Características do financiamento das pesquisas no âmbito do Proadi-SUS}

De 2009 a 2014, foram investidos R\$ 66.494.000,00 em 46 pesquisas realizadas por seis hospitais de excelência, cinco dos quais localizados na região Sudeste; e um, no Sul. O volume de recursos alocados em cada projeto variou de $\mathrm{R} \$ 294.941,00$, a $\mathrm{R} \$$ 6.330.466,00, com uma média de $\mathrm{R} \$ 1.445 .521,00$ por projeto (tabela 1). Foram identificados projetos relacionados com 12 temáticas, 10 das quais são médicas, com predomínio de pesquisas em cardiologia e oncologia que juntas representaram $63.8 \%$ do investimento no período.

Tabela 1. Distribuição de projetos de pesquisa financiados pelo Proadi-SUS e investimentos, segundo área temática, no período de 2009 a 2014. Decit, MS

\begin{tabular}{lrrr}
\hline \multirow{2}{*}{ Área Temática } & Número de projetos & Investimento (Milhões de reais) \\
\cline { 3 - 4 } Cardiologia & 23 & № & \% \\
Oncologia & 9 & 25,3 & 38,1 \\
Neurologia & 2 & 17,1 & 25,7 \\
Terapia celular & 1 & 6,8 & 10,2 \\
Endocrinologia & 2 & 6,3 & 9,5 \\
Pneumologia & 1 & 3,5 & 3,3 \\
Nefrologia & 3 & 2,1 & 2,7 \\
Saúde mental & 1 & 1,8 & 2,7 \\
Análise sócio territorial & 1 & 1,8 & 0,8 \\
Hematologia & 1 & 0,6 & 0,8 \\
Saneamento básico & 1 & 0,6 & 0,4 \\
Assistência farmacêutica & 1 & 0,3 & 0,4 \\
\hline Total & $\mathbf{4 6}$ & $\mathbf{1 0 0 0}$ \\
\hline
\end{tabular}

Fonte: Relatórios de prestação de contas dos projetos Proadi. 
Foram financiados no período ensaios clínicos (25), estudos de coorte (12), caso controle (1), estudos transversais (6) e ecológicos (2); $59 \%$ dos projetos desenvolveram estudos de avaliação de tecnologias em saúde. Predominaram ensaios clínicos randomizados com $60,2 \%$ do investimento total ( $\mathrm{R} \$ 40$ milhões), seguidos dos estudos de coorte com $27,1 \%$ dos recursos ( $\$ 18$ milhões). Os estudos de menor frequência de investimento foram os de caso controle e transversais/ecológicos, no total de 2,4\% do investimento ( $\mathrm{R} \$ 8,4$ milhões). Quando considerado o tipo de pesquisa, a média de investimento nos ensaios clínicos foi de R \$ 1,6 milhão por projeto; nos estudos de coorte, $\mathrm{R} \$ 1,5$ milhão; casos controle, 1,3 milhão; seguidos dos estudos transversais com R\$ 1 milhão e dos estudos ecológicos com R\$ 450 mil.

Os hospitais de excelência coordenaram 18 estudos multicêntricos nas áreas de cardiologia, oncologia, neurologia, nefrologia, medicina intensiva e hematologia. Esses estudos foram conduzidos com protocolo único, proposto pelo hospital de excelência, e realizados em colaboração com centros de pesquisa nacionais e internacionais.

\section{Impacto das pesquisas na dimensão avanços no conhecimento}

\section{CATEGORIA ATIVIDADE DE PESQUISA}

A análise de impacto identificou 28 projetos (60,8\% do total) nos quais foram verificadas as produções técnicas e científicas e participações em eventos para disseminação dos resultados.

Foram identificadas 60 publicações, em 41,3\% dos projetos financiados, entre artigos científicos, resumos em anais de eventos científicos, dissertações, capítulos de livros (tabela2). Predominaram os artigos científicos $(65,0 \%)$. Outras produções, como relato de caso e nota técnica, foram identificadas em menor número. Verificou-se que 23 publicações fizeram referência ao Ministério da Saúde e/ou ao Proadi como financiadores, e 15 citaram a Fundação de Amparo à Pesquisa (FAP), 0 Conselho Nacional de Desenvolvimento Científico e Tecnológico (CNPq), o próprio hospital ou outras instituições nacionais e internacionais. As demais (22) não mencionaram as instituições de financiamento. Nos 39 artigos publicados, as áreas temáticas foram cardiologia, oncologia, hematologia, medicina intensiva, neurologia e terapia celular. Aárea com maior proporção de artigos científicos foi cardiologia $(61,5 \%)$.

Tabela 2. Tipos de publicações científicas por número e proporção de projetos de pesquisa financiados pelo Proadi, no período de 2009 a 2014. Decit, MS

\begin{tabular}{lrrrr}
\hline \multirow{2}{*}{ Tipo de publicação } & \multicolumn{2}{c}{ Publicações } & Projetos de pesquisa com publicaçõesa \\
\cline { 2 - 4 } & № & $\%$ & № & \% \\
\hline Artigo científico & 39 & 65,0 & 17 & 37,0 \\
Resumo expandido em periódico e anais de eventos & 3 & 5,0 & 3 & 10,9 \\
científicos & 11 & 18,3 & 1 & 2,5 \\
Resumo em periódico e anais de eventos científicos & 1 & 1,7 & 4 & 8,7 \\
Dissertação de mestrado & 4 & 6,7 & 2 & 4,4 \\
Outras publicações & 2 & 3,3 & & \\
Capítulo de livro & 60 & 100 & & \\
\hline Total de publicações & & & & \\
\hline
\end{tabular}

Fonte: Relatórios de prestação de contas dos projetos Proadi.

a - Total de projetos $=46$. 


\section{QUALIDADE DA PESQUISA}

O fator de impacto (JCR), o H-index da base de dados Scopus e a classificação Qualis Capes foram identificados em 39 periódicos, nacionais (9) e internacionais (30), indexados. Seis periódicos tinham fator de impacto acima de 3 ; 19 (48,7\%) apresentaram classificação Qualis Capes entre A1 e A2, e os demais (51,3\%), entre B1 e B3. Apenas um periódico não foi classificado pelo sistema Qualis Capes. Os artigos científicos foram veiculados em periódicos internacionais (76,9\%), dos quais Estados Unidos (30,7\%) e Reino Unido (25,6\%) foram os de maior frequência.

Os 39 artigos produzidos foram ranqueados pelo somatório das citações nas bases de dados Scopus e Google acadêmico, e os 10 artigos mais citados (quadro 1) referem-se às áreas de nefrologia, cardiologia, hematologia e medicina intensiva. Esses artigos receberam entre 32 e 242 citações e foram publicados em periódicos cujas sedes internacionais situam-se em países norte-americanos e europeus.

Quadro 1. Ranking dos dez artigos científicos mais citados, por título, periódico e ano de publicação, no período de 2009 a 2014. Decit, MS

\begin{tabular}{|c|c|c|c|c|c|}
\hline & Título do artigo & Periódico & Ano & Scopus & $\begin{array}{l}\text { Google } \\
\text { acadêmico }\end{array}$ \\
\hline 1 & $\begin{array}{l}\text { Acetylcysteine for prevention of renal outcomes in patients undergoing coro- } \\
\text { nary and peripheral vascular angiography: main results from the randomized } \\
\text { acetylcysteine for contrast induced nephropathy trial (ACT) }\end{array}$ & $\begin{array}{l}\text { Circulation (2011), Sep 13; 124(11) } \\
1250-9\end{array}$ & 2011 & 209 & 242 \\
\hline 2 & $\begin{array}{l}\text { Is a pre- operative brain natriuretic peptide or } \mathrm{N} \text {-terminal pro-B-Type natri- } \\
\text { uretic peptide measurements an independent predictor of adverse cardiovas- } \\
\text { cular outcomes with } 30 \text { days of noncardiac surgery? A systematic review and } \\
\text { meta-analysis of observational studies }\end{array}$ & $\begin{array}{l}\text { Journal of the American College of } \\
\text { Cardiology, (2009) Vol 54, N. } 17 .\end{array}$ & 2009 & 133 & 201 \\
\hline 3 & $\begin{array}{l}\text { Myeloablative reduced-toxicity IV busulfan-fludarabine and allogenic hema- } \\
\text { topoietic stem cell transplant in the sixth through eight decades of life }\end{array}$ & $\begin{array}{l}\text { Biol. Blood Marrow Transplant } \\
\text { (2011), October; } 17(10): 1490-1496\end{array}$ & 2011 & 69 & 82 \\
\hline 4 & $\begin{array}{l}\text { Brazilian urban population genetic structure reveals a high degree of admix- } \\
\text { ture }\end{array}$ & $\begin{array}{l}\text { European Journal of Genetics (2012), } \\
20,111-116 \text {. }\end{array}$ & 2012 & 50 & 79 \\
\hline 5 & $\begin{array}{l}\text { Effects of alveolar recruitment maneuvers on clinical outcomes in patients } \\
\text { with acute respiratory distress syndrome: a systematic review and meta- } \\
\text { analysis }\end{array}$ & $\begin{array}{l}\text { Intensive Care Medicine (2014), vol. } \\
40, \text { p. } 1227-1240,2014\end{array}$ & 2014 & 40 & 64 \\
\hline 6 & $\begin{array}{l}\text { High sensitivity troponin T concentrations in patients undergoing noncardiac } \\
\text { surgery: a prospective cohort study }\end{array}$ & $\begin{array}{l}\text { Clinical Biochemistry (2011), 44, } \\
\text { 1021-1024 }\end{array}$ & 2011 & 41 & 59 \\
\hline 7 & $\begin{array}{l}\text { The role of pancreatic iron stores assessed by magnetic resonance imaging } \\
(\mathrm{MRI}) \text { in beta thalassemic patients }\end{array}$ & $\begin{array}{l}\text { European Journal of Radiology } 81 \\
\text { (2012) } 1465-1470\end{array}$ & 2012 & 22 & 30 \\
\hline 8 & $\begin{array}{l}\text { Cardiovascular simulator improvement Pressure versus volume loop assess- } \\
\text { ment }\end{array}$ & Artificial Organs (2011), Vol. 35, n.5 & 2011 & 15 & 24 \\
\hline 9 & $\begin{array}{l}\text { Effect of lung recruitment and titrated Positive End-Expiratory Pressure } \\
\text { (PEEP) vs low PEEP on mortality in patients with acute respiratory distress } \\
\text { syndrome - A randomized clinical trial }\end{array}$ & $\begin{array}{l}\text { JAMA October 10, } 2017 \text { Vol. 318, } \\
\text { n. } 14\end{array}$ & 2017 & 18 & 15 \\
\hline 10 & $\begin{array}{l}\text { Association between pre-operative statin use and major cardiovascular com- } \\
\text { plications among patients undergoing non-cardiac surgery: the vision study }\end{array}$ & $\begin{array}{l}\text { European Heart journal (2016) 37, } \\
177-185\end{array}$ & 2016 & 32 & 0 \\
\hline
\end{tabular}

Nota: O ranking foi realizado com base na soma das citações das bases Scopus e Google acadêmico. Acesso em abril de 2018.

\section{DISSEMINAC̣ÃO DE RESULTADOS}

A partir do levantamento de dados, foi possível identificar que em 26 dos 46 projetos
(56,5\%) os resultados das pesquisas foram divulgados em eventos (tabela 3). Entre as 85 participações, 24 foram apresentações orais, e 12 pôsteres. Em 49 participações, não foram 
identificadas as modalidades de apresentação. O maior percentual de participações $(38,8 \%)$ ocorreu em congressos científicos. Também foram identificadas participações de pesquisadores em eventos não científicos como programas de TV e entrevistas na mídia (sítios, jornal, campanha, matérias em revistas e jornais não científicos). Dois projetos receberam prêmios (um na categoria inovação em gestão estadual e outro de melhor trabalho publicado em evento científico).

Tabela 3. Participação em eventos científicos por número e proporção de projetos de pesquisa financiados pelo Proadi, no período de 2009 a 2014. Decit, MS

\begin{tabular}{|c|c|c|c|c|}
\hline \multirow[t]{2}{*}{ Evento } & \multicolumn{2}{|c|}{ Participação em evento } & \multicolumn{2}{|c|}{$\begin{array}{l}\text { Projetos de pesquisa com } \\
\text { participação em evento }\end{array}$} \\
\hline & № & $\%$ & № & $\%$ \\
\hline Congresso & 33 & 38,8 & 19 & 41,3 \\
\hline Seminário & 1 & 1,2 & 1 & 2,2 \\
\hline Encontro & 15 & 17,6 & 11 & 23,9 \\
\hline Conferência & 9 & 10,6 & 5 & 10,9 \\
\hline Simpósio & 5 & 5,9 & 4 & 8,7 \\
\hline $\begin{array}{l}\text { Outros eventos científicos (mostra, oficina, } \\
\text { fórum, reunião, mesa redonda, jornada) }\end{array}$ & 12 & 14,1 & 10 & 21,7 \\
\hline Eventos não científicos & 10 & 11,8 & 5 & 10,9 \\
\hline Total & 85 & 100 & & \\
\hline
\end{tabular}

Fonte: Relatórios de prestação de contas dos projetos Proadi.

a - Total de projetos $=46$.

\section{Discussões}

Este estudo mostrou que, no período de 2009 a 2014, foram aplicados por meio de renúncia fiscal (Proadi-SUS) R\$ 66,49 milhões em 46 pesquisas. $\mathrm{O}$ investimento se concentrou nas áreas de cardiologia e oncologia (63,8\%), evidenciando a importância das Doenças Crônicas Não Transmissíveis (DCNT) no cenário de transição epidemiológica vivenciado no País, e a preocupação com o impacto que esses agravos têm na economia e no sistema público de saúde. A atenção às doenças crônicas representa desafio constante ao sistema de saúde, no que tange à capacidade diagnóstica e à necessidade de maior densidade tecnológica para o tratamento. Nesse sentido, o financiamento de pesquisas que respondam às necessidades do SUS pode contribuir para melhorar a resolutividade nos níveis de atenção ${ }^{15}$.
No mesmo período, os investimentos governamentais com pesquisa em saúde pelo Programa Pesquisa para o SUS (PPSUS) somaram aproximadamente R 192 milhões em 1.796 pesquisas. Destes, R\$ 23,7 milhões foram aplicados para o desenvolvimento de 373 pesquisas clínicas em doenças cônicas não transmissíveis, com investimento médio de R \$ 63.6 mil por projeto. A média de recursos por projeto de pesquisa clínica no Proadi (R \$ 1,4 milhão) é considerada alta, em contraste com os valores aplicados pelo PPSUS.

No Proadi, ao comparar o investimento por tipo de pesquisa, verificou-se que $60,2 \%$ dos recursos ( $\mathrm{R} \$ 40$ milhões) foram direcionados a financiar pesquisas clínicas em DCNT, em contraste com $12,3 \%$ quando considerada a mesma subagenda e tipo e pesquisa pelo programa PPSUS. O enfrentamento das DCNT demanda estudos longitudinais que avancem na compreensão dos determinantes socioeconômicos, de 
raça/etnia e culturais associados à progressão desses agravos na população brasileira. Apesar da recorrência de doenças transmissíveis, em especial as arboviroses no País, não foram financiadas pelo Proadi no Decit pesquisas nessa subagenda. Diante do impacto da morbidade e da mortalidade de doenças transmissíveis, ações conjuntas de vigilância, mobilização social e em pesquisa e desenvolvimento de métodos diagnósticos efetivos devem nortear os esforços e recursos governamentais para a pesquisa ${ }^{16}$.

O predomínio de ensaios clínicos randomizados $(60,2 \%)$ indicou uma tendência ao financiamento de estudos clínicos que avaliaram métodos e estratégias terapêuticas com potencial de subsidiar a tomada de decisão em saúde. Na escala hierárquica dos delineamentos, tradicionalmente, os ensaios clínicos randomizados contribuem para o melhor nível de evidência, sobretudo em questões de ordem clínica e de modo geral, demandam maior aporte de recursos, inclusive financeiros e tempo para execução ${ }^{17,18}$, fato que possivelmente explica a maior frequência desses delineamentos nas pesquisas financiadas, além da capacidade e qualificação das instituições executoras, consideradas de excelência pelo Ministério da Saúde. Intervenções testadas em ensaios clínicos podem contribuir para adoção de melhores práticas em saúde, com resultados para os usuários do sistema de saúde. Para promover melhores resultados em saúde, estudos também apontam para a tomada de decisão compartilhada, com o envolvimento e a participação do usuário no processo decisório ${ }^{19}$.

Sob o ponto de vista da qualidade da evidência, os ensaios clínicos randomizados vinham sendo considerados ao longo dos anos, pela literatura, como o padrão ouro para análises de intervenções terapêuticas. Entretanto, recentemente, estudos têm mostrado que algumas limitações (ausência de validade externa, tempo de seguimento insuficiente para detectar efeitos de longo prazo e incapacidade de avaliar efeitos individualizados do tratamento) associadas aos ensaios clínicos interferem em sua aplicabilidade, sobretudo no âmbito das decisões em saúde pública, que frequentemente exigem tomada de decisão urgente, como nos casos de surtos e epidemias por doenças transmissíveis. Embora possam gerar evidências de qualidade, fundamentais para a validação das decisões de ordem clínica, novas abordagens observacionais em estudos de avaliação da implementação têm sido utilizadas como fonte de evidência decisiva para a gestão ${ }^{20}$.

O reduzido número de estudos epidemiológicos $(2,4 \%)$ no conjunto de projetos financiados no período mostrou uma lacuna, dada a relevância da epidemiologia translacional no contexto da saúde pública brasileira. Evidências obtidas a partir desses estudos subsidiam o planejamento e a implementação de programas e políticas direcionadas à prevenção e ao controle dos determinantes de saúde tanto em nível individual como coletivo21.

O mapeamento da produção científica evidenciou que $41,3 \%$ dos projetos avaliados gerou produtos tangíveis (outputs) em áreas de relevância para saúde pública. Ao considerar o quantitativo de projetos apoiados e o volume de recursos investidos, esse percentual é considerado baixo, em relação a outros estudos da literatura ${ }^{8}$. Nos 28 projetos $(60,8 \%)$ em que foram identificados produtos, os artigos mais citados, de modo geral, avaliaram tecnologias e produziram evidências que, agregadas ao conhecimento global, podem ser utilizadas nos níveis individual (atitudes), interpessoal (influência), coletivo e nas organizações para produção de novos conhecimentos, para a tomada de decisão de gestores e profissionais de saúde, contribuindo para a indução de mudanças na prática clínica e na política.

A participação de pesquisadores em eventos científicos (41,3\%) indica que os resultados das pesquisas foram disseminados entre a comunidade científica, favorecendo o intercâmbio e a troca de experiências entre os pesquisadores. Observa-se também que a divulgação de resultados (11,8\%) em meios não científicos, como programas de televisão e matérias em revistas, demonstra o alcance do conhecimento gerado como informação à sociedade, revelando a potencial influência para além do meio acadêmico. Entretanto, merece destaque o predomínio da 
divulgação de resultados, sobretudo no meio acadêmico, quando há a necessidade de ampliar o conhecimento produzido a diferentes públicos, principalmente para gestores de saúde. Se os projetos têm coerência com a transição epidemiológica, as ações dos pesquisadores poderiam também orientar-se para influenciar a tomada de decisão dos níveis gerenciais, individuais, familiares, comunitários e na sociedade. Nesse sentido, estratégias não apenas de divulgação aos diferentes públicos, mas também de implementação de resultados podem contribuir para o aprimoramento do Proadi-SUS e qualificação do sistema de saúde 22 .

No tocante à produtividade e à participação em eventos científicos, estudos conduzidos no Reino Unido ${ }^{23}$, Canadá ${ }^{24}$, Hong Kong ${ }^{25}$ e Brasil ${ }^{8}$ encontraram maior volume de produtos gerados em pesquisas com financiamento público, em contraste com os resultados deste estudo, cuja produtividade pode ser considerada baixa. Sob ponto de vista da avaliação, os sistemas universitários de pesquisas nacionais e internacionais adotam como critério a produtividade técnico científica, e, com isso, aumentam a competividade entre os pesquisadores na corrida por publicações em periódicos de alto impacto, favorecendo a disseminação de resultados, condição fundamental para o avanço do conhecimento ${ }^{26}$.

Em países como Reino Unido, em que o sistema de saúde é público, pesquisas também são financiadas por instituições filantrópicas, que aportam recursos para o desenvolvimento produtivo, inovação, comercialização de medicamentos e de outras tecnologias para uso na área da saúde. Estudos apontam que o retorno desse investimento excede a taxa anual de retorno esperada pelo governo britânico, quando considerados investimentos públicos de modo geral. No contexto brasileiro, a renúncia fiscal concedida aos hospitais de excelência fundamenta-se na lógica de estímulo ao aprimoramento do SUS. Do mesmo modo, espera-se que esses investimentos gerem produtos que possam reduzir o abismo tecnológico nacional, beneficiando os pacientes do sistema ${ }^{27}$.

É importante destacar que a atribuição das evidências de uma pesquisa aos impactos de longo prazo, como mudanças em indicadores de saúde, benefícios econômicos e sociais, é um processo complexo e não linear, sendo influenciado pelo contexto político, econômico e pelos diversos atores envolvidos ${ }^{9}$.

O desenvolvimento de modelos de avaliação de impacto é relativamente recente, e abordagens baseadas no modelo Payback, com modificações, têm fornecido informações relevantes em relação à translação do conhecimento, como retorno ao investimento aplicado na pesquisa. Métodos e ferramentas capazes de associar o conhecimento gerado e sua utilização (translação do conhecimento) contribuem para a compreensão do impacto dos resultados da pesquisa ${ }^{\mathbf{2 8}}$. Entendese que é importante fomentar conhecimento e assegurar a disseminação e compartilhamento, visando apoiar processos que permitam construir novos conhecimentos, instrumentos e práticas capazes de gerar inovação e melhorias à saúde.

Contudo, reduzir a lacuna entre as evidências de pesquisa e a prática permanece como um desafio aos sistemas de saúde, e ignorar essa questão retarda o acesso aos usuários e profissionais da atenção às melhores evidências e tratamentos mais eficazes. Portanto, é necessária a construção de mecanismos que melhorem a utilização das evidências na política e na prática. Iniciativas como o fomento à pesquisa translacional com enfoque na produção de evidências e de soluções aos problemas prioritários dos serviços de saúde têm sido adotadas por alguns sistemas internacionais de saúde. Nesse processo, ações como o envolvimento de gestores, usuários e profissionais ligados aos diferentes níveis de atenção, nas pesquisas financiadas, além do estímulo às parcerias entre as organizações, potencialmente geram oportunidades para o alcance de melhores resultados ao sistema. O engajamento dos diferentes atores na elaboração das agendas, desenvolvimento e realização da pesquisa torna o processo participativo e contribui para que os resultados possam ser implementados ${ }^{29}$.

A interação entre os gestores no âmbito da política e pesquisadores favorece a troca de conhecimentos e potencializa a influência das 
evidências na política. $\mathrm{O}$ acesso oportuno à evidência de qualidade e a disseminação das pesquisas são considerados elementos facilitadores no processo de absorção dos resultados de pesquisa na política, na tomada de decisão em saúde e embasam novos estudos. Contudo, no âmbito da gestão, há que se considerar que os aspectos metodológicos da pesquisa e o uso de linguagem científica podem representar desafios a serem superados, uma vez que exigem corpo técnico qualificado e especializado para interpretação das evidências. Nessa perspectiva, alguns estudos reportam que estratégias, como a utilização de agentes ou intermediários que promovem a conexão entre a pesquisa e os diferentes públicos (Knowledge brokers), e ferramentas de tradução do conhecimento podem contribuir para apropriação dos achados científicos pelos sistemas de saúde. Ações e estratégias direcionadas à translação do conhecimento poderiam ser discutidas e incorporadas ao programa Proadi-SUS, a fim de potencializar a utilização das evidências produzidas no sistema de saúde22,30,31.

\section{Considerações finais}

Os R \$ 66,49 milhões de reais aplicados nas pesquisas do Proadi-SUS resultaram em publicações científicas (65\%) publicadas em periódicos de alto impacto, que contribuíram para a disseminação das evidências produzidas no meio acadêmico (56,5\%), favorecendo o desenvolvimento de novos estudos e a apropriação dos resultados pelos sistemas de saúde. Em que pese a reduzida proporção de produtos tangíveis (41,3\%), no conjunto de projetos financiados no período, potencialmente, houve avanço no conhecimento produzido em relação às DCNT. Contudo, permanece uma lacuna, a ser estudada, no que diz respeito à transferência e ao uso desses conhecimentos pela gestão para o aprimoramento do sistema. O Proadi foi criado com o propósito de apoiar o SUS. Nesse sentido, estudos que busquem avaliar a aplicação das evidências na construção de novos conhecimentos, na prática clínica e na gestão podem contribuir para a compreensão da medida do impacto dessas pesquisas em outras dimensões.

Este estudo buscou identificar o impacto das pesquisas financiadas pelo Proadi sob o ponto de vista dos avanços do conhecimento. Entretanto, apresenta algumas limitações, como: i) a análise de indicadores em apenas uma das cinco dimensões propostas pelo modelo CAHS, o que não permite inferir o impacto dessas pesquisas em relação à transferência de conhecimentos, tomada de decisão informada, benefícios ao setor saúde e à economia, sendo necessários estudos mais aprofundados que abordem as demais dimensões desse modelo; ii) a principal fonte de dados desta pesquisa foi o relatório de prestação de contas elaborado pelas instituições participantes, o que possibilita que essas instituições demonstrem preferencialmente resultados positivos. Eventuais produtos primários e secundários gerados após a apresentação dos relatórios de prestação de contas podem não ter sido considerados neste estudo, uma vez que efeitos de médio e longo prazo nem sempre são facilmente mensuráveis e demandam estudos mais robustos; iii) a classificação dos projetos segundo área temática possivelmente limitou a verificação dos resultados esperados. Este estudo evidenciou a importância do aprimoramento dos mecanismos de gestão da pesquisa pelo Decit.

\section{Colaboradores}

Moraes LH (0000-0003-1994-2180)* contribuiu para a concepção e elaboração do manuscrito, análise e interpretação dos dados e finalização do artigo. Angulo-Tuesta (00000002-3231-5918)* colaborou para a interpretação dos dados e revisão crítica do artigo. Funghetto SS (0000-0002-9332-9029)* contribuiu para o delineamento do artigo. Rehem TCMSB (0000-0002-4491-1661)* participou do delineamento do artigo, revisão crítica e aprovação da versão final do manuscrito. 


\section{Referências}

1. Greenhalgh T, Raftery J, Hanney S, et al. Research impact: a narrative review. BMC Med. 2016; 14(1):78.

2. Pang T, Sadana R, Hanney S, et al. Policy and Practice Knowledge for better health - a conceptual framework and foundation for health research systems. Bull World Health Organ. [internet]. 2003 [acesso em 2019 mar 8]; 81(11):815-820. Disponível em: https:// www.ncbi.nlm.nih.gov/pubmed/14758408.

3. Hanney SR, González Block MA. Building health research systems to achieve better health. Health Res Policy Sys [internet]. 2006 [acesso em 2019 fev 9]; 4(10):16. Disponível em: https://health-policy-systems. biomedcentral.com/articles/10.1186/1478-4505-4-10.

4. Science D. The nature, scale and beneficiaries of research impact; 2015. p. 28-38.

5. Tenório M, Mello GA, Viana ALD. Políticas de fomento à ciência, tecnologia e inovação em saúde no Brasil e o lugar da pesquisa clínica. Ciênc. Saúde Colet. 2017; 22(5):1441-54.

6. Brasil. Lei $\mathrm{n}^{0} 12.101$, de 27 de novembro de 2009. Dispõe sobre a certificação das entidades beneficentes de assistência social; regula os procedimentos de isenção de contribuições para a seguridade social [internet]. Brasília, DF; 2009. [acesso em 2018 nov 5]. Disponível em: http://www.planalto.gov.br/ccivil_03/_Ato2007-2010/2009/Lei/L12101.htm.

7. Banzi R, Moja L, Pistotti V, et al. Conceptual frameworks and empirical approaches used to assess the impact of health research: an overview of reviews. Health Res Policy Syst. 2011; (26):1-10.

8. Angulo-Tuesta A, Santos LMP. Evaluation of the impact of maternal and neonatal morbidity and mortality research funded by the Ministry of Health in Brazil. Research Evaluation [internet]. 2015 [acesso em 2018 nov 8]; 24:355-368. Disponível em: https://academic. oup.com/rev/article-abstract/24/4/355/1525348.

9. Canadian Academy of Health Sciences. Making an
Impact: Panel on return on investment in health research. Making and impact: A preferred framework and indicators to measure returns on investment in health research. Ottawa: Canadian Academy of Health Sciences; 2009.

10. Angulo-Tuesta A, Santos LMP, Natalizi DA. Impacto da pesquisa em saúde nos avanços de conhecimento, construção de capacidades de pesquisa e políticas informadas por evidências: Um estudo de caso sobre a morbimortalidade materna no Brasil. São Paulo Med J. 2016; 134(2):153-62.

11. Graham KER, Chorzempa HL, Valentine PA, et al. Evaluating health research impact : Development and implementation of the Alberta Innovates - Health Solutions impact framework. Res Eval. 2012; (21):354-67.

12. Pons JM V, Adam P, Solans-dome M. Assessment of the impact of a clinical and health services research call in Catalonia. Res Eval. 2012; (21):319-28.

13. Nason E, Curran B, Hanney S, et al. Evaluating health research funding in Ireland: Assessing the impacts of the health research board of Ireland's funding activities. Res Eval. 2011; 20(3):193-200.

14. Angulo-Tuesta A. Impactos das Pesquisas sobre Morbimortalidade Materna e Neonatal Financiadas pelo Ministério da Saúde. [tese]. Brasília, DF: Universidade de Brasília; 2015.

15. Guimarães RM, Andrade SSCA, Machado EL, et al. Diferenças regionais na transição da mortalidade por doenças cardiovasculares no Brasil, 1980 a 2012. 2015; 37(2):83-9.

16. Teixeira MG, Nascimento C. Conquistas do SUS no enfrentamento das doenças transmissíveis The achievements of the SUS in tackling the communicable diseases. 2018; 23(6):1819-1828.

17. Devereaux PJ, Yusuf S. The evolution of the randomized controlled trial and its role in evidence-based 
decision making. J Intern Med. 2003; 254:105-13.

18. Nedel WL, Silveira F. Different research designs and their characteristics in intensive care. Rev Bras Ter Intensiva. 2016; 28(3):256-60.

19. Alegria M, Nakash O, Johnson K, et al. Effectiveness of the DECIDE Interventions on Shared Decision Making and Perceived Quality of Care in Behavioral Health With Multicultural Patients A Randomized Clinical Trial. JAMA Psychiatry. 2018; 75(4):325-35.

20. Jeffrey M, Drazen MD, David P, et al. Evidence for Health Decision Making - Beyond Randomized, Controlled Trials. N Engl J Med. [internet]. 2017 [acesso em 2019 ago 3]; 465-475. Disponível em: https:// www.nejm.org/doi/pdf/10.1056/NEJMral614394.

21. Szklo M. Epidemiologia translacional: algumas considerações. Epidemiol e Serv. Saúde. 2015; 24(1):10315

22. Angulo-Tuesta A, Santos LMP, Iturri JA. Processos e desafios da interação entre pesquisa e política na perspectiva dos pesquisadores. Ciênc. Saúde Colet. 2018; 23(1):7-15.

23. Kuruvilla S, Mays N, Pleasant A, et al. Describing the impact of health research: A Research Impact Framework. BMC Health Serv Res. 2006; 6(134):1-18.

24. Caddell AJ, Hatchette JE, Mcgrath PJ. Examining the impact of health research facilitated by small peer-reviewed research operating grants in a women's and children's health centre. BCM res. notes. [internet]. 2010 [acesso em 2019 fev 9]; 3(107). Disponível em: https://bmcresnotes .biomedcentral.com/track/ pdf/10.1186/1756-0500-3-107.

25. Kwan P, Johnston J, Fung AYK, et al. A systematic evaluation of payback of publicly funded health and health services research in Hong Kong. BMC Health Serv Res. 2007; 7(121):1-10.

26. Iriarte JAB, Deslandes SF, Martin D, et al. A avaliação da produção científica nas subáreas da Saúde Coletiva: limites do atual modelo e contribuições para o debate. Cad. Saúde Pública. 2015; 31(10):2137-2147.

27. Sussex J, Feng Y, Mestre-Ferrandiz J, et al. Quantifying the economic impact of government and charity funding of medical research on private research and development funding in the United Kingdom. BMC Med [internet]. 2016 [acesso em 2019 fev 9]; 14(1). Disponível em: http://dx.doi.org/10.1186/s12916-0160564-z.

28. Searles A, Doran C, Attia J, et al. An approach to measuring and encouraging research translation and research impact. Heal Res Policy Syst. 2016; 14(1):1-13.

29. Rose S, Milat A, Penna A, et al. Translational Research Grants Scheme (TRGS): a new approach to strengthening health system research capacity. Public Heal Res Pract. 2018; 28(3):1-5.

30. Grimshaw JM, Eccles MP, Lavis JN, et al. Knowledge translation of research findings. Implement Sci. 2012; 7:50.

31. Oliver K, Innvar S, Lorenc T, et al. A systematic review of barriers to and facilitators of the use of evidence by policymakers. BMC Health Serv Res. [internet]. 2014 [acesso em 2019 fev 6]; 14(2). Disponível em: https://bmchealthservres.biomedcentral.com/ articles/10.1186/1472-6963-14-2.

Recebido em 31/03/2019

Aprovado em 21/08/2019

Conflito de interesses: inexistente

Suporte financeiro: não houve 